\title{
RATE OF CONVERGENCE OF BOUNDED VARIATION FUNCTIONS BY A BÉZIER-DURRMEYER VARIANT OF THE BASKAKOV OPERATORS
}

\author{
VIJAY GUPTA and ULRICH ABEL
}

Received 6 May 2002

We consider a Bézier-Durrmeyer integral variant of the Baskakov operators and study the rate of convergence for functions of bounded variation.

2000 Mathematics Subject Classification: 41A36, 41A25, 26A45.

1. Introduction. Let $W(0, \infty)$ be the class of functions $f$ which are locally integrable on $(0, \infty)$ and are of polynomial growth as $t \rightarrow \infty$, that is, for some positive $r$, there holds $f(t)=O\left(t^{r}\right)$ as $t \rightarrow \infty$. The Durrmeyer variant $\tilde{V}_{n}$ of the Baskakov operators associates to each function $f \in W(0, \infty)$ the series

$$
\tilde{V}_{n}(f ; x)=(n-1) \sum_{k=0}^{\infty} p_{n, k}(x) \int_{0}^{\infty} p_{n, k}(t) f(t) d t, \quad x \in[0, \infty),
$$

where

$$
p_{n, k}(x)=\left(\begin{array}{c}
n+k-1 \\
k
\end{array}\right) x^{k}(1+x)^{-n-k}
$$

is the Baskakov basis function. Note that (1.1) is well defined, for $n \geq r+2$, provided that $f(t)=O\left(t^{r}\right)$ as $t \rightarrow \infty$. The operators (1.1) were first introduced by Sahai and Prasad [9]. They termed these operators as modified Lupaş operators. In 1991, Sinha et al. [10] improved and corrected the results of [9] and denoted $\tilde{V}_{n}$ as modified Baskakov operators. The rate of convergence of the operators (1.1) on functions of bounded variation was studied in $[8,11]$.

We mention that Agrawal and Thamer [2] considered the variant

$$
M_{n}(f ; x)=(n-1) \sum_{k=1}^{\infty} p_{n, k}(x) \int_{0}^{\infty} p_{n, k-1}(t) f(t) d t+(1+x)^{-n} f(0)
$$

of the operators (1.1) and studied its properties in subsequent papers [3, 4, 5]. See also [1]. The rate of convergence of the operators discussed by Agrawal and Thamer was studied by the first author in [7]. 
For each function $f \in W(0, \infty)$ and $\alpha \geq 1$, we consider the Bézier-type BaskakovDurrmeyer operators $\tilde{V}_{n, \alpha}$ as

$$
\tilde{V}_{n, \alpha}(f ; x)=(n-1) \sum_{k=0}^{\infty} Q_{n, k}^{(\alpha)}(x) \int_{0}^{\infty} p_{n, k}(t) f(t) d t,
$$

where

$$
\begin{aligned}
Q_{n, k}^{(\alpha)}(x) & =J_{n, k}^{\alpha}(x)-J_{n, k+1}^{\alpha}(x), \\
J_{n, k}(x) & =\sum_{j=k}^{\infty} p_{n, j}(x)
\end{aligned}
$$

It is obvious that $\tilde{V}_{n, \alpha}$ are positive linear operators and $\tilde{V}_{n, \alpha}(1 ; x)=1$. In the special case $\alpha=1$, the operators $\widetilde{V}_{n, \alpha}$ reduce to the operators $\widetilde{V}_{n} \equiv \widetilde{V}_{n, 1}$. Some basic properties of $J_{n, k}$ are as follows:

(i) $J_{n, k}(x)-J_{n, k+1}(x)=p_{n, k}(x)(k=0,1,2, \ldots)$;

(ii) $J_{n, k}^{\prime}(x)=n p_{n+1, k-1}(x)(k=1,2,3, \ldots)$;

(iii) $J_{n, k}(x)=n \int_{0}^{x} p_{n+1, k-1}(t) d t(k=1,2,3, \ldots)$;

(iv) $0<\cdots<J_{n, k+1}(x)<J_{n, k}(x)<\cdots<J_{n, 1}(x)<J_{n, 0}(x) \equiv 1(x>0)$;

(v) $J_{n, k}$ is strictly increasing on $[0, \infty)$.

In this paper, we study the rate of convergence for the new sequence of operators (1.4), for functions $f$ of bounded variation. Our result essentially generalizes and improves the results of $[8,11]$. Furthermore, we find the limit of the sequence $\tilde{V}_{n, \alpha}(f ; x)$ for bounded locally integrable functions $f$ having a discontinuity of the first kind at $x \in(0, \infty)$.

2. The main results. As a main result, we derive the following estimate on the rate of convergence.

THEOREM 2.1. Assume that $f \in W(0, \infty)$ is a function of bounded variation on every finite subinterval of $(0, \infty)$. Furthermore, let $\alpha \geq 1, \lambda>2$, and $x \in(0, \infty)$ be given. Then, for each $r \in \mathbb{N}$, there exists a constant $M(f, \alpha, r, x)$ such that for sufficiently large $n$, the Bézier-type Baskakov-Durrmeyer operators $\tilde{V}_{n, \alpha}$ satisfy the estimate

$$
\begin{aligned}
& \left|\tilde{V}_{n, \alpha}(f ; x)-\left[\frac{1}{\alpha+1} f(x+)+\frac{\alpha}{\alpha+1} f(x-)\right]\right| \\
& \leq \frac{\alpha(10+11 x)}{2 \sqrt{n x(1+x)}|f(x+)-f(x-)|} \\
& \quad+\frac{2 \alpha \lambda(1+x)+x}{n x} \sum_{k=1}^{n} \bigvee_{x-x / \sqrt{k}}^{x+x / \sqrt{k}}\left(g_{x}\right)+\frac{M(f, \alpha, r, x)}{n^{r}},
\end{aligned}
$$

where

$$
g_{x}(t)= \begin{cases}f(t)-f(x-) & (0 \leq t<x) \\ 0 & (t=x) \\ f(t)-f(x+) & (x<t<\infty)\end{cases}
$$

and $\bigvee_{a}^{b}\left(g_{x}\right)$ is the total variation of $g_{x}$ on $[a, b]$ 
REMARK 2.2. The exponent $r$ in the $O$-term of (2.1) can be chosen arbitrary large.

As an immediate consequence of Theorem 2.1, we obtain in the special case $\alpha=1$ the following estimate which improves the results of $[8,11]$.

COROLLARY 2.3. Under the assumptions of Theorem 2.1, there holds, for sufficiently large $n$,

$$
\begin{aligned}
& \left|\tilde{V}_{n}(f ; x)-\frac{1}{2}[f(x+)+f(x-)]\right| \\
& \quad \leq \frac{(10+11 x)}{2 \sqrt{n x(1+x)}}|f(x+)-f(x-)| \\
& \quad+\frac{2 \lambda(1+x)+x}{n x} \sum_{k=1}^{n} \bigvee_{x-x / \sqrt{k}}^{x+x / \sqrt{k}}\left(g_{x}\right)+\frac{M(f, 1, r, x)}{n^{r}},
\end{aligned}
$$

where $g_{x}$ is defined as in Theorem 2.1.

THEOREM 2.4. Let $x \in(0, \infty)$. If $f \in L(0, \infty)$ has a discontinuity of the first kind at $x$, then

$$
\lim _{n \rightarrow \infty} \tilde{V}_{n, \alpha}(f ; x)=\frac{1}{\alpha+1} f(x+)+\frac{\alpha}{\alpha+1} f(x-) .
$$

3. Auxiliary results. In order to prove our main result, we will need the following lemmas. Throughout the paper, for each real $x$, let $\psi_{x}(t)=t-x$.

LEMMA 3.1 (see [6]). Let $\left\{\xi_{i}\right\}_{i=1}^{\infty}$ be a sequence of independent and identically distributed random variables with finite variance such that the expectation $E\left(\xi_{i}\right)=a_{1} \in$ $\mathbb{R} \equiv(-\infty, \infty)$, and the variance $V\left(\xi_{i}\right)=b_{1}^{2}>0$. Assume that $E\left|\xi_{i}-a_{1}\right|^{3}<\infty$. Then there exists a constant $c$ with $1 / \sqrt{2 \pi}<c<0.82$ such that, for all $n=1,2,3, \ldots$ and all $t \in \mathbb{R}$,

$$
\left|P\left(\frac{1}{b_{1} \sqrt{n}} \sum_{k=1}^{n}\left(\xi_{i}-a_{1}\right) \leq t\right)-\frac{1}{\sqrt{2 \pi}} \int_{-\infty}^{t} e^{-u^{2} / 2} d u\right| \leq c \frac{E\left|\xi_{i}-a_{1}\right|^{3}}{\sqrt{n} b_{1}^{3}} .
$$

LEMMA 3.2 (see [10]). For each fixed $x \in[0, \infty)$ and $m \in \mathbb{N}_{0}$, the central moments $\tilde{V}_{n}\left(\psi_{x}^{m} ; x\right)$ of the Baskakov-Durrmeyer operators (1.1) satisfy

$$
\tilde{V}_{n}\left(\psi_{x}^{m} ; x\right)=O\left(n^{-\lfloor(m+1) / 2\rfloor}\right) \quad(n \rightarrow \infty) .
$$

In particular,

$$
\tilde{V}_{n}(1 ; x)=1, \quad \tilde{V}_{n}\left(\psi_{x}^{2} ; x\right)=\frac{2(n-1) x(1+x)}{(n-2)(n-3)}+\frac{2(1+2 x)^{2}}{(n-2)(n-3)} .
$$

REMARK 3.3. Note that, given any $\lambda>2$ and any $x>0$, for all $n$ sufficiently large, we have the estimate

$$
\tilde{V}_{n}\left(\psi_{x}^{2} ; x\right)<\frac{\lambda x(1+x)}{n}
$$


LEMMA 3.4 (see [13]). For all $x>0$ and $n, k \in \mathbb{N}$, there holds

$$
J_{n, k}^{\alpha}(x) p_{n, k}(x) \leq Q_{n, k}^{(\alpha)}(x) \leq \alpha p_{n, k}(x)<\frac{\alpha \sqrt{1+x}}{\sqrt{2 e n x}} .
$$

Throughout, let

$$
\begin{aligned}
& K_{n, \alpha}(x, t)=(n-1) \sum_{k=0}^{\infty} Q_{n, k}^{(\alpha)}(x) p_{n, k}(t), \\
& \lambda_{n, \alpha}(x, y)=\int_{0}^{y} K_{n, \alpha}(x, t) d t .
\end{aligned}
$$

With this definition, for each function $f \in W(0, \infty)$, there holds, for all sufficiently large $n$,

$$
\tilde{V}_{n, \alpha}(f ; x)=\int_{0}^{\infty} K_{n, \alpha}(x, t) f(t) d t
$$

Note that, in particular,

$$
\lambda_{n, \alpha}(x, \infty)=\int_{0}^{\infty} K_{n, \alpha}(x, u) d u=1 .
$$

LEMMA 3.5. For each $\lambda>2$ and, for all sufficiently large $n$, there exist, for all $x \in$ $(0, \infty)$,

$$
\begin{aligned}
\lambda_{n, \alpha}(x, y) & =\int_{0}^{y} K_{n, \alpha}(x, t) d t \leq \frac{\lambda \alpha x(1+x)}{n(x-y)^{2}} \quad(0 \leq y<x), \\
1-\lambda_{n, \alpha}(x, z) & =\int_{z}^{\infty} K_{n, \alpha}(x, t) d t \leq \frac{\lambda \alpha x(1+x)}{n(z-x)^{2}} \quad(x<z<\infty) .
\end{aligned}
$$

Proof. First we prove (3.10). There holds

$$
\begin{aligned}
\int_{0}^{y} K_{n, \alpha}(x, t) d t & \leq \int_{0}^{y} K_{n, \alpha}(x, t) \frac{(x-t)^{2}}{(x-y)^{2}} d t \\
& \leq(x-y)^{-2} \tilde{V}_{n, \alpha}\left(\psi_{x}^{2} ; x\right) \\
& \leq \alpha(x-y)^{-2} \tilde{V}_{n, 1}\left(\psi_{x}^{2} ; x\right),
\end{aligned}
$$

where we applied Lemma 3.4. Now (3.10) is a consequence of Remark 3.3. The proof of (3.11) is similar.

LEMMA 3.6 (see [13]). Let $\left\{\xi_{i}\right\}_{i=1}^{\infty}$ be a sequence of independent random variables with the same geometric distribution

$$
P\left(\xi_{1}=k\right)=\left(\frac{x}{1+x}\right)^{k} \frac{1}{1+x} \quad(k \in \mathbb{N}),
$$

where $x>0$ is a parameter. Then,

$$
E\left(\xi_{1}\right)=x, \quad E\left(\xi_{1}-E \xi_{1}\right)^{2}=x(1+x), \quad E\left|\xi_{1}-E \xi_{1}\right|^{3} \leq 3 x(1+x)^{2} .
$$


LEMMA 3.7. For all $x \in(0, \infty)$ and $k=0,1,2, \ldots$, there hold

$$
\begin{aligned}
\left|J_{n, k}^{\alpha}(x)-J_{n-1, k+1}^{\alpha}(x)\right| & \leq \frac{\alpha(10+11 x)}{2 \sqrt{n x(1+x)}}, \\
\left|J_{n, k}^{\alpha}(x)-J_{n-1, k}^{\alpha}(x)\right| & \leq \frac{\alpha(10+11 x)}{2 \sqrt{n x(1+x)}} .
\end{aligned}
$$

Proof. First we prove (3.15). Proceeding along the lines of [8, Lemma 2.8] and [12], it is easily checked that

$$
\begin{aligned}
\left|J_{n, k}(x)-J_{n-1, k+1}(x)\right| & \leq \frac{2(0.82) E\left|\xi_{1}-E \xi_{1}\right|^{3}}{\sqrt{n}(x(1+x))^{3 / 2}}+\frac{x}{\sqrt{2 \pi n x(1+x)}} \\
& \leq \frac{2(0.82) \cdot 3 x(1+x)^{2}}{\sqrt{n}(x(1+x))^{3 / 2}}+\frac{x}{2 \sqrt{n x(1+x)}} \\
& \leq \frac{10+11 x}{2 \sqrt{n x(1+x)}},
\end{aligned}
$$

where we used Lemmas 3.1 and 3.6. Application of the inequality $\left|a^{\alpha}-b^{\alpha}\right| \leq \alpha|a-b|$, for $0 \leq a, b \leq 1$, and $\alpha \geq 1$ yields (3.15). The proof of (3.16) is similar.

\section{Proofs of the main results}

Proof OF Theorem 2.1. Our starting point is the identity

$$
\begin{aligned}
f(t)= & \frac{1}{\alpha+1} f(x+)+\frac{\alpha}{\alpha+1} f(x-)+\left(\operatorname{sign}(t-x)+\frac{\alpha-1}{\alpha+1}\right) \frac{f(x+)-f(x-)}{2} \\
& +g_{x}(t)+\delta_{x}(t)\left(f(x)-\frac{f(x+)+f(x-)}{2}\right)
\end{aligned}
$$

where $\delta_{x}(t)=1(t=x)$ and $\delta_{x}(t)=0(t \neq x)$ (see [12, Equation (28)]). Since $\tilde{V}_{n, \alpha}\left(\delta_{x} ; x\right)=$ 0 , we conclude that

$$
\begin{aligned}
& \left|\tilde{V}_{n, \alpha}(f ; x)-\left[\frac{1}{\alpha+1} f(x+)+\frac{\alpha}{\alpha+1} f(x-)\right]\right| \\
& \quad \leq \frac{1}{2}\left|\tilde{V}_{n, \alpha}(\operatorname{sign}(t-x) ; x)+\frac{\alpha-1}{\alpha+1}\right||f(x+)-f(x-)|+\left|\tilde{V}_{n, \alpha}\left(g_{x} ; x\right)\right| .
\end{aligned}
$$

First, we obtain

$$
\begin{aligned}
\tilde{V}_{n, \alpha}(\operatorname{sign}(t-x) ; x) & =(n-1) \sum_{j=0}^{\infty} Q_{n, j}^{(\alpha)}(x)\left(\int_{x}^{\infty} p_{n, j}(t) d t-\int_{0}^{x} p_{n, j}(t) d t\right) \\
& =(n-1) \sum_{j=0}^{\infty} Q_{n, j}^{(\alpha)}(x)\left(\int_{0}^{\infty} p_{n, j}(t) d t-2 \int_{0}^{x} p_{n, j}(t) d t\right) \\
& =1-2(n-1) \sum_{j=0}^{\infty} Q_{n, j}^{(\alpha)}(x) \int_{0}^{x} p_{n, j}(t) d t .
\end{aligned}
$$


Using

$$
\sum_{j=0}^{k} p_{n-1, j}(x)=(n-1) \int_{x}^{\infty} p_{n, k}(t) d t
$$

we conclude that

$$
\begin{aligned}
\tilde{V}_{n, \alpha}(\operatorname{sign}(t-x) ; x) & =1-2 \sum_{j=0}^{\infty} Q_{n, j}^{(\alpha)}(x)\left(1-\sum_{k=0}^{j} p_{n-1, k}(x)\right) \\
& =-1+2 \sum_{k=0}^{\infty} p_{n-1, k}(x) \sum_{j=k}^{\infty} Q_{n, j}^{(\alpha)}(x) \\
& =-1+2 \sum_{k=0}^{\infty} p_{n-1, k}(x) J_{n, k}^{\alpha}(x)
\end{aligned}
$$

since $\sum_{j=0}^{\infty} Q_{n, j}^{(\alpha)}(x)=1$. Therefore, we obtain

$$
\tilde{V}_{n, \alpha}(\operatorname{sign}(t-x) ; x)+\frac{\alpha-1}{\alpha+1}=2 \sum_{k=0}^{\infty} p_{n-1, k}(x) J_{n, k}^{\alpha}(x)-\frac{2}{\alpha+1} \sum_{k=0}^{\infty} Q_{n-1, k}^{(\alpha+1)}(x)
$$

since $\sum_{k=0}^{\infty} Q_{n-1, k}^{(\alpha+1)}(x)=1$. By the mean value theorem, it follows that

$$
Q_{n-1, k}^{(\alpha+1)}(x)=J_{n-1, k}^{\alpha+1}(x)-J_{n-1, k+1}^{\alpha+1}(x)=(\alpha+1) p_{n-1, k}(x) \gamma_{n, k}^{\alpha}(x),
$$

where $J_{n-1, k+1}(x)<\gamma_{n, k}(x)<J_{n-1, k}(x)$. Hence,

$$
\tilde{V}_{n, \alpha}(\operatorname{sign}(t-x) ; x)+\frac{\alpha-1}{\alpha+1}=2 \sum_{k=0}^{\infty} p_{n-1, k}(x)\left(J_{n, k}^{\alpha}(x)-\gamma_{n, k}^{\alpha}(x)\right),
$$

where

$$
J_{n, k}^{\alpha}(x)-J_{n-1, k}^{\alpha}(x)<J_{n, k}^{\alpha}(x)-\gamma_{n, k}^{\alpha}(x)<J_{n, k}^{\alpha}(x)-J_{n-1, k+1}^{\alpha}(x) .
$$

Lemma 3.7 implies that

$$
\left|\tilde{V}_{n, \alpha}(\operatorname{sign}(t-x) ; x)+\frac{\alpha-1}{\alpha+1}\right| \leq \frac{\alpha(10+11 x)}{\sqrt{n x(1+x)}} \quad \text { for } x \in(0, \infty) .
$$

In order to complete the proof of the theorem, we need an estimate of $\tilde{V}_{n, \alpha}\left(g_{x} ; x\right)$. We use the integral representation (3.8) and decompose $[0, \infty)$ into three parts as follows:

$$
\begin{aligned}
\tilde{V}_{n, \alpha}\left(g_{x} ; x\right) & =\left(\int_{0}^{x-x / \sqrt{n}}+\int_{x-x / \sqrt{n}}^{x+x / \sqrt{n}}+\int_{x+x / \sqrt{n}}^{\infty}\right) K_{n, \alpha}(x, t) g_{x}(t) d t \\
& =I_{1}+I_{2}+I_{3}, \quad \text { say. }
\end{aligned}
$$

We start with $I_{2}$. For $t \in[x-x / \sqrt{n}, x+x / \sqrt{n}]$, we have

$$
\left|g_{x}(t)\right| \leq \bigvee_{x-x / \sqrt{n}}^{x+x / \sqrt{n}}\left(g_{x}\right)
$$


and therefore

$$
\left|I_{2}\right| \leq \bigvee_{x-x / \sqrt{n}}^{x+x / \sqrt{n}}\left(g_{x}\right) \leq \frac{1}{n} \sum_{k=1}^{n} \bigvee_{x-x / \sqrt{k}}^{x+x / \sqrt{k}}\left(g_{x}\right)
$$

Next we estimate $I_{1}$. Let $y=x-x / \sqrt{n}$. Using integration by parts with (3.7), we have

$$
I_{1}=\int_{0}^{y} g_{x}(t) d_{t} \lambda_{n, \alpha}(x, t)=g_{x}(y) \lambda_{n, \alpha}(x, y)-\int_{0}^{y} \lambda_{n, \alpha}(x, t) d_{t} g_{x}(t)
$$

Since $\left|g_{x}(y)\right|=\left|g_{x}(y)-g_{x}(x)\right| \leq \bigvee_{y}^{x}\left(g_{x}\right)$, we conclude that

$$
\left|I_{1}\right| \leq \bigvee_{y}^{x}\left(g_{x}\right) \lambda_{n, \alpha}(x, y)+\int_{0}^{y} \lambda_{n, \alpha}(x, t) d_{t}\left(-\bigvee_{t}^{x}\left(g_{x}\right)\right)
$$

Since $y=x-x / \sqrt{n} \leq x,(3.10)$ implies that

$$
\left|I_{1}\right| \leq \frac{\lambda \alpha x(1+x)}{n(x-y)^{2}} \bigvee_{y}^{x}\left(g_{x}\right)+\frac{\lambda \alpha x(1+x)}{n} \int_{0}^{y} \frac{1}{(x-t)^{2}} d_{t}\left(-\bigvee_{t}^{x}\left(g_{x}\right)\right)
$$

Integrating the last term by parts, we get

$$
\left|I_{1}\right| \leq \frac{\lambda \alpha x(1+x)}{n}\left(x^{-2} \bigvee_{0}^{x}\left(g_{x}\right)+2 \int_{0}^{y} \frac{\bigvee_{t}^{x}\left(g_{x}\right)}{(x-t)^{3}} d t\right)
$$

Replacing the variable $y$ in the last integral by $x-x / \sqrt{n}$, we obtain

$$
\begin{aligned}
\int_{0}^{x-x / \sqrt{n}} \bigvee_{t}^{x}\left(g_{x}\right)(x-t)^{-3} d t & =\sum_{k=1}^{n-1} \int_{x / \sqrt{k+1}}^{x / \sqrt{k}} \bigvee_{x-t}^{x}\left(g_{x}\right) t^{-3} d t \\
& \leq \frac{1}{2 x^{2}} \sum_{k=1}^{n} \bigvee_{x-x / \sqrt{k}}^{x}\left(g_{x}\right) .
\end{aligned}
$$

Hence,

$$
\left|I_{1}\right| \leq \frac{2 \lambda \alpha(1+x)}{n x} \sum_{k=1}^{n} \bigvee_{x-x / \sqrt{k}}^{x}\left(g_{x}\right)
$$

Finally, we estimate $I_{3}$. We let

$$
\tilde{g}_{x}(t)= \begin{cases}g_{x}(t) & (0 \leq t \leq 2 x) \\ g_{x}(2 x) & (2 x<t<\infty)\end{cases}
$$


and divide $I_{3}=I_{31}+I_{32}$, where

$$
\begin{aligned}
& I_{31}=\int_{x+x / \sqrt{n}}^{\infty} K_{n, \alpha}(x, t) \tilde{g}_{x}(t) d t, \\
& I_{32}=\int_{2 x}^{\infty} K_{n, \alpha}(x, t)\left[g_{x}(t)-g_{x}(2 x)\right] d t .
\end{aligned}
$$

With $y=x+x / \sqrt{n}$, the first integral can be written in the form

$$
\begin{gathered}
I_{31}=\lim _{R \rightarrow+\infty}\left\{g_{x}(y)\left[1-\lambda_{n, \alpha}(x, y)\right]+\tilde{g}_{x}(R)\left[\lambda_{n, \alpha}(x, R)-1\right]\right. \\
\left.+\int_{y}^{R}\left[1-\lambda_{n, \alpha}(x, t)\right] d_{t} \tilde{g}_{x}(t)\right\} .
\end{gathered}
$$

By (3.11), we conclude that

$$
\begin{aligned}
\left|I_{31}\right| & \leq \frac{\lambda \alpha x(1+x)}{n} \lim _{R \rightarrow+\infty}\left\{\frac{\bigvee_{x}^{y}\left(g_{x}\right)}{(y-x)^{2}}+\frac{\left|\tilde{g}_{x}(R)\right|}{(R-x)^{2}}+\int_{y}^{R} \frac{1}{(t-x)^{2}} d_{t}\left(\bigvee_{x}^{t}\left(\tilde{g}_{x}\right)\right)\right\} \\
& =\frac{\lambda \alpha x(1+x)}{n}\left\{\frac{\bigvee_{x}^{y}\left(g_{x}\right)}{(y-x)^{2}}+\int_{y}^{2 x} \frac{1}{(t-x)^{2}} d_{t}\left(\bigvee_{x}^{t}\left(g_{x}\right)\right)\right\} .
\end{aligned}
$$

In a similar way as above we obtain

$$
\int_{y}^{2 x} \frac{1}{(t-x)^{2}} d_{t}\left(\bigvee_{x}^{t}\left(g_{x}\right)\right) \leq x^{-2} \bigvee_{x}^{2 x}\left(g_{x}\right)-\frac{\bigvee_{x}^{y}\left(g_{x}\right)}{(y-x)^{2}}+x^{-2} \sum_{k=1}^{n-1} \bigvee_{x}^{x+x / \sqrt{k}}\left(g_{x}\right)
$$

which implies the estimate

$$
\left|I_{31}\right| \leq \frac{2 \lambda \alpha(1+x)}{n x} \sum_{k=1}^{n} \bigvee_{x}^{x+x / \sqrt{k}}\left(g_{x}\right)
$$

We proceed with $I_{32}$. By assumption, there exists an integer $r$ such that $f(t)=O\left(t^{2 r}\right)$ as $t \rightarrow \infty$. Thus, for a certain constant $M>0$, depending only on $f, x$, and $r$, we have

$$
\begin{aligned}
\left|I_{32}\right| & \leq M(n-1) \sum_{k=0}^{\infty} Q_{n, k}^{(\alpha)}(x) \int_{2 x}^{\infty} p_{n, k}(t) t^{2 r} d t \\
& \leq \alpha M(n-1) \sum_{k=0}^{\infty} p_{n, k}(x) \int_{2 x}^{\infty} p_{n, k}(t) t^{2 r} d t,
\end{aligned}
$$

where we used Lemma 3.4. Obviously, $t \geq 2 x$ implies $t \leq 2(t-x)$ and it follows that

$$
\left|I_{32}\right| \leq 2^{2 r} \alpha M(n-1) \sum_{k=0}^{\infty} p_{n, k}(x) \int_{0}^{\infty} p_{n, k}(t)(t-x)^{2 r} d t=2^{2 r} \alpha M \tilde{V}_{n}\left(\psi_{x}^{2 r} ; x\right)
$$


By Lemma 3.2, the central moments of the Baskakov-Durrmeyer operators (1.1) satisfy $\tilde{V}_{n}\left(\psi_{x}^{2 r} ; x\right)=O\left(n^{-r}\right)(n \rightarrow \infty)$, and we obtain

$$
I_{32}=O\left(n^{-r}\right) \quad(n \rightarrow \infty) .
$$

Collecting the estimates (4.13), (4.19), (4.25), and (4.28) yields with regard to (4.11)

$$
\left|\tilde{V}_{n, \alpha}\left(g_{x} ; x\right)\right| \leq \frac{2 \lambda \alpha(1+x)+x}{n x} \sum_{k=1}^{n} \bigvee_{x-x / \sqrt{k}}^{x+x / \sqrt{k}}\left(g_{x}\right)+O\left(n^{-r}\right) \quad(n \rightarrow \infty)
$$

Finally, combining (4.2), (4.10), and (4.29), we obtain (2.1). This completes the proof of Theorem 2.1.

Proof of Theorem 2.4. Since the function $\psi_{x}^{2}$ given by $\psi_{x}^{2}(t)=(t-x)^{2}$ is of bounded variation on every finite subinterval of $[0, \infty)$, we deduce from Theorem 2.1 that, for all $x \in(0, \infty)$,

$$
\lim _{n \rightarrow \infty} \tilde{V}_{n, \alpha}\left(\psi_{x}^{2} ; x\right)=0
$$

If $f \in L_{\infty}(0, \infty)$, then $g_{x}$ defined as in (2.2) is also bounded and is continuous at the point $x$. By the Korovkin theorem, we conclude that

$$
\lim _{n \rightarrow \infty} \tilde{V}_{n, \alpha}\left(g_{x} ; x\right)=g_{x}(x)=0 .
$$

Therefore, the right-hand side of inequality (4.2) tends to zero as $n \rightarrow \infty$. This completes the proof of Theorem 2.4.

ACKNOWLEDGMENT. The authors are thankful to the referee for the valuable comments that helped to improve this paper. Moreover, the referee provided further important references.

\section{REFERENCES}

[1] P. N. Agrawal and A. J. Mohammad, On convergence of derivatives of a new sequence of linear positive operators, to appear in Rev. Un. Mat. Argentina.

[2] P. N. Agrawal and K. J. Thamer, Approximation of unbounded functions by a new sequence of linear positive operators, J. Math. Anal. Appl. 225 (1998), no. 2, 660-672.

[3] __ Inverse theorem for a new sequence of linear positive operators on $L_{p}$-spaces, Rev. Un. Mat. Argentina 42 (2000), no. 1, 1-8.

[4] _ A new sequence of linear positive operators for higher order $L_{p}$-approximation, Rev. Un. Mat. Argentina 41 (2000), no. 4, 9-18.

[5] _ Degree of approximation by a new sequence of linear operators, Kyungpook Math. J. 41 (2001), no. 1, 65-73.

[6] W. Feller, An Introduction to Probability Theory and Its Applications. Vol. II, John Wiley \& Sons, New York, 1966.

[7] V. Gupta, Rate of approximation by a new sequence of linear positive operators, Comput. Math. Appl. 45 (2003), no. 12, 1895-1904.

[8] V. Gupta and G. S. Srivastava, An estimate of the rate of convergence of modified Baskakov operators for functions of bounded variation, Kyungpook Math. J. 36 (1996), no. 2, 237-247. 
[9] A. Sahai and G. Prasad, On simultaneous approximation by modified Lupas operators, J. Approx. Theory 45 (1985), no. 2, 122-128.

[10] R. P. Sinha, P. N. Agrawal, and V. Gupta, On simultaneous approximation by modified Baskakov operators, Bull. Soc. Math. Belg. Sér. B 43 (1991), no. 2, 217-231.

[11] Y. Wang and S. Guo, Rate of approximation of functions of bounded variation by modified Lupas operators, Bull. Austral. Math. Soc. 44 (1991), no. 2, 177-188.

[12] X.-M. Zeng and W. Chen, On the rate of convergence of the generalized Durrmeyer type operators for functions of bounded variation, J. Approx. Theory 102 (2000), no. 1, $1-12$.

[13] X.-M. Zeng and V. Gupta, Rate of convergence of Baskakov-Bézier type operators for locally bounded functions, Comput. Math. Appl. 44 (2002), no. 10-11, 1445-1453.

Vijay Gupta: School of Applied Sciences, Netaji Subhas Institute of Technology, Azad Hind Fauj Marg, Sector-3, Dwarka, New Delhi-110045, India

E-mail address: vijay@nsit.ac. in

Ulrich Abel: Fachbereich MND, Fachhochschule Giessen-Friedberg, University of Applied Sciences, Wilhelm-Leuschner-Straße 13, 61169 Friedberg, Germany

E-mail address: U1rich. Abe1@mnd.fh-friedberg.de 


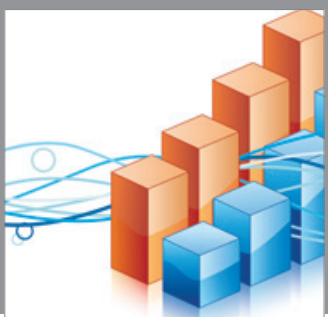

Advances in

Operations Research

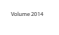

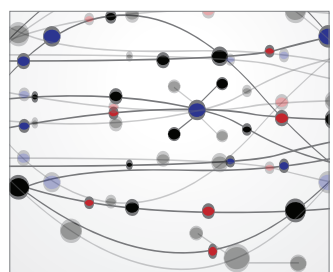

\section{The Scientific} World Journal
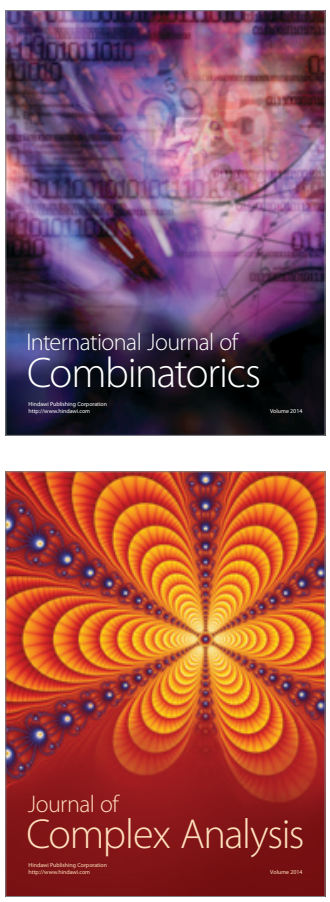

International Journal of

Mathematics and

Mathematical

Sciences
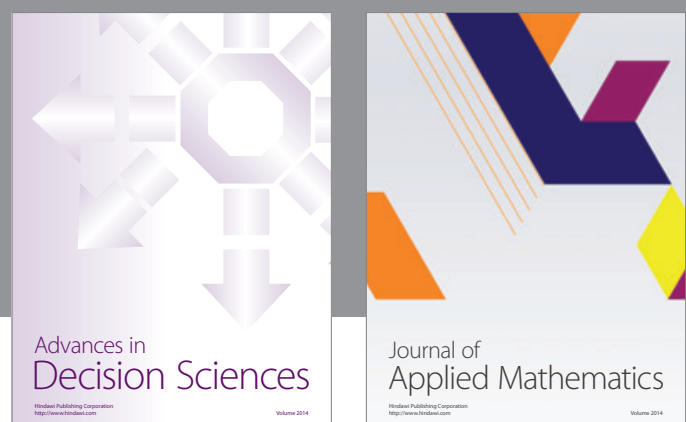

Journal of

Applied Mathematics
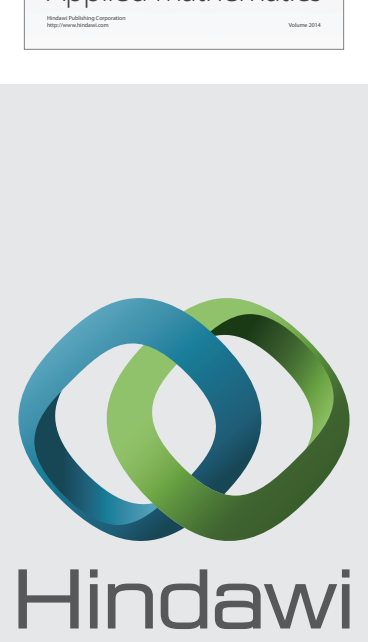

Submit your manuscripts at http://www.hindawi.com
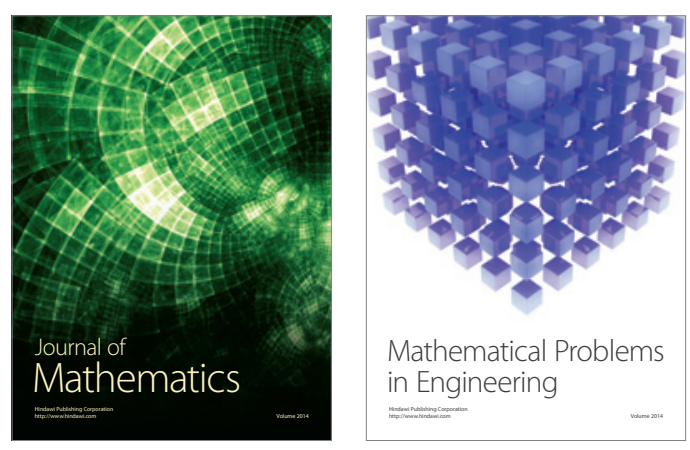

Mathematical Problems in Engineering
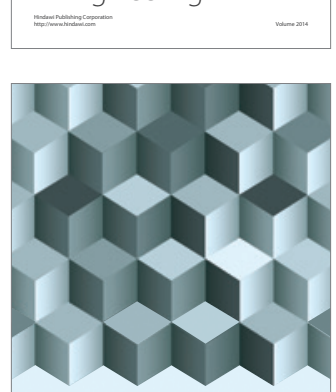

Journal of

Function Spaces
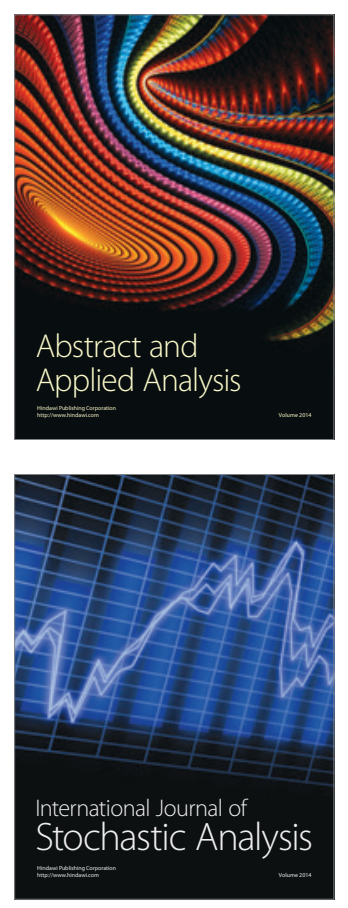

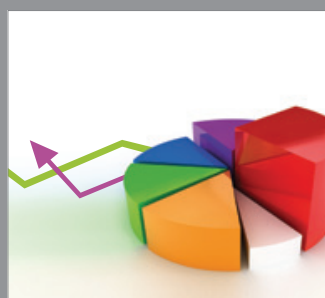

ournal of

Probability and Statistics

Promensencen
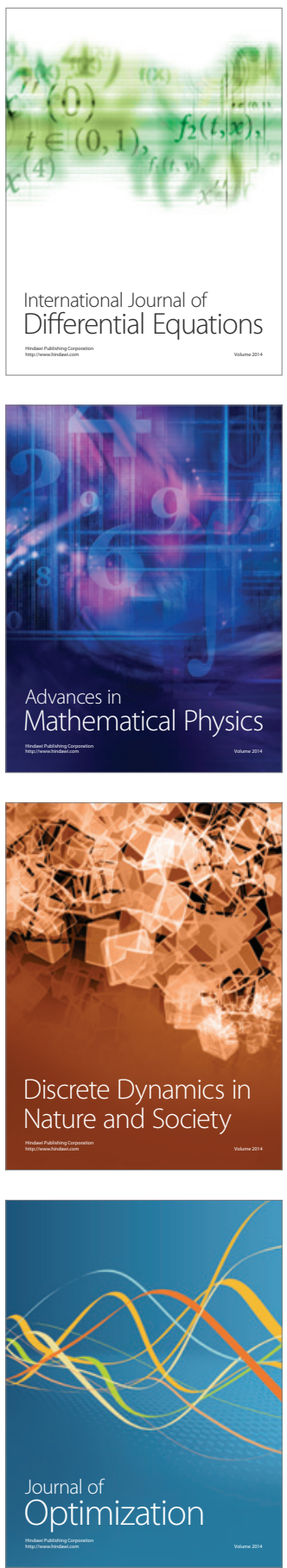